\title{
Neue Leitlinien zu Thrombose, Lungenembolie und PAVK
}

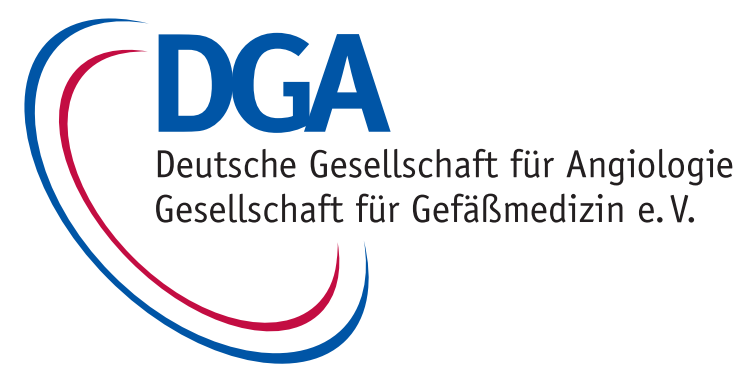

Die Deutschen Gesellschaft für Angiologie (DGA) sieht in der Erarbeitung von Leitlinien eine Kernaufgabe. Sie sollen dazu beitragen, dass die alles andere als zufriedenstellende Versorgungsqualität verbessert wird - und zwar auf interdisziplinärer Ebene. Auf der DGA-Jahrestagung wurden zwei unter der Federführung der DGA überarbeitete Leitlinien vorgestellt. Sie sollen noch im Oktober publiziert werden.

\section{S2-Leitlinie zur Diagnostik und Therapie der Venenthrombose und Lungenembolie}

Die DGA hat zusammen mit 15 Fachgesellschaften die interdisziplinäre S2Leitlinie zur Diagnostik und Therapie der Venenthrombose und der Lungenembolie aktualisiert. Hier die wesentlichen Botschaften, zusammengestellt von der DGA:

-Eine erste Diagnose kann durch den Hausarzt durch Erhebung der klinischen Wahrscheinlichkeit und einen D-Dimer-Test erfolgen. Wenn die klinische Wahrscheinlichkeit niedrig und der D-Dimer-Test, der altersabhängig zu befunden ist, negativ sind, ist eine Thrombose ausgeschlossen.

- Andernfalls müssen Patienten an einen Spezialisten verwiesen werden, der anhand eines kompletten Ultraschall des Beins eine Thrombose diagnostizieren kann.
_Für die Behandlung der Thrombose und Lungenembolie stehen zusätzlich zur konventionellen Therapie mit Heparin und Vitamin-K-Antagonisten auch die sogenannten direkten oder neuen Antikoagulanzien (DOAC/ NOAC) zur Verfügung.

_ Die Behandlungsdauer mit Medikamenten ist von Patient zu Patient sehr unterschiedlich und hängt von verschiedenen Risikofaktoren z. B. einer Rezidivthrombose, Thrombose nach einem Unfall oder ohne Ursache, Blutungsrisiko usw. ab.

\section{S3-Leitlinie: Periphere Arterielle Verschlusskrankheit - Diagnostik und Therapie}

Die S3-Leitlinie zur Diagnostik und Therapie der peripheren arteriellen Verschlusskrankheit (PAVK) wurde in $\mathrm{Zu}$ sammenarbeit mit über 20 weiteren Fachgesellschaften aktualisiert.

\section{Karotisstenosen: OP oder Stent?}

Wie in der interventionellen Kardiologie hat in der Angiologie etwas zeitversetzt der Wettlauf um die besten Prothesen, Stents und Ballone begonnen. Die Studienlage ist dürftig, Vergleichsprüfungen mit langen Laufzeiten sind rar. Neue Daten gab es nun zur Therapie der Karotisstenosen.

n der Vorbeugung und Therapie von Karotis bedingten Schlaganfällen war die Karotis-Thrombendarteriektomie (CEA) lange das einzige effektive operative Verfahren. Seit wenigen Jahren versuchen sich auch die Interventionalisten an den Karotisstenosen mit diversen Stent-Systemen (CAS).

Wann welche Methode wie häufig eingesetzt wird, hat nun eine Analyse aus Daten des Deutschen Instituts zur Qualitätssicherung in der Medizin unter- sucht. Ausgewertet wurden Daten des BQS-Instituts für Qualität und Patientensicherheit aus den Jahren 2003 bis 2008 und des Folgeinstituts AQUA (Institut für angewandte Qualitätsförderung und Forschung im Gesundheitswesen) von 2009 bis 2014 .

Die noch unveröffentlichten Ergebnisse diskutierte der Gefäßchirurg Prof. Hans-Henning Eckstein vom Klinikum rechts der Isar der Technischen Universität München.
_ Die adäquate Diagnostik und Behandlung von PAVK-Patienten wird aufgrund stetig steigender Patientenzahlen immer wichtiger. Die Untersuchung einer Krankenkasse hat ergeben, dass bei der Hälfte der Amputationen, die wegen einer PAVK durchgeführt werden, keine ausreichende bildgebende Diagnostik im Vorfeld stattgefunden hat.

- Auch deshalb ist eine enge Zusammenarbeit von Angiologen, Diabetologen, Nephrologen und Gefäßchirurgen unerlässlich.

_Inzwischen können viele Patienten mit einem endovaskulären Eingriff behandelt werden, die Zahl der Operationen ist dagegen leicht rückläufig. -Die Nachsorge durch regelmäßige Kontrollen bei einem Facharzt (z. B. Angiologen) hilft, bei erneut auftretenden Problemen schnell und gezielt reagieren zu können.

(J.A.)
Erste Überraschung: In über 650 deutschen Kliniken werden diese Eingriffe (CEA oder CAS) angeboten - mehr als vermutet wurde. Die endovaskuläre Stentimplantation in die Karotiden wird seit 2012 erfasst, die CEA-Statistik beginnt 2003. Während sich bei den CEA ein langsamer Anstieg von 19.355 im Jahre 2003 auf 26.802 im Vorjahr ergab, der sich seit etwa sechs Jahren auf ein Plateau eingependelt hat, blieb die Anzahl der CAS annähernd konstant: 6.172 in 2012, $6.054 \mathrm{im}$ Vorjahr.

Von einem explosiven Anstieg der Implantationen von Karotisstents wie auch der operativen Eingriffe kann also keine Rede sein.

Die Altersverteilung zeigt die erwarteten Trends. Das Kollektiv der CEA-Patienten im Alter von 70 bis 79 nimmt 